\title{
Development and validation of a triplex qPCR assay to detect efflux pump-mediated antibiotic resistance in Burkholderia pseudomallei
}

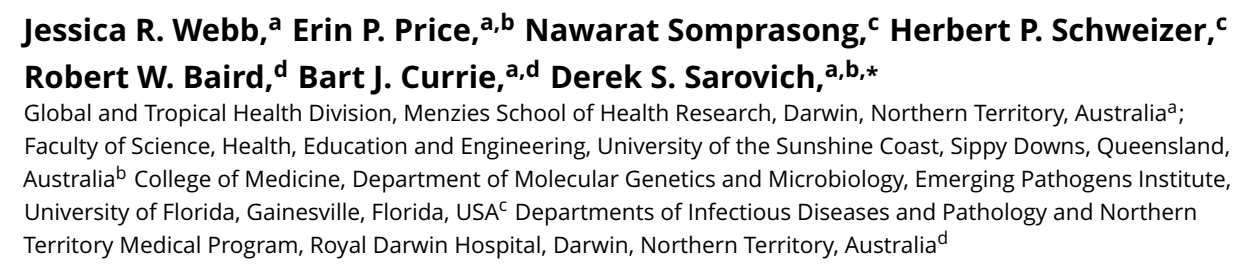

ABSTRACT Burkholderia pseudomallei, the causative agent of the deadly tropical disease melioidosis, is intrinsically resistant to many antibiotics, leaving few effective treatment options. Trimethoprim-sulfamethoxazole (SXT), meropenem (MEM) and doxycycline (DOX) are valuable antibiotics for melioidosis treatment due to inherently low or no primary resistance. Although considered rare, upregulation of one or more resistance-nodulation-division (RND) efflux pumps is now known to lead to acquired resistance towards these drugs in B. pseudomallei. Here, we developed a triplex quantitative PCR assay to detect upregulation of the three clinically relevant RND efflux systems: AmrAB-OprA, BpeB-OprB and BpeEF-OprC. The triplex assay was tested on seven clinically-derived B. pseudomallei isogenic pairs, where the latter strain of each pair had altered regulator activity and exhibited reduced susceptibility to SXT, MEM or DOX. The triplex assay accurately detected efflux pump upregulation between isogenic pairs, which corresponded with decreased antibiotic susceptibility. We further verified assay performance on eight laboratory-generated B. pseudomallei mutants encoding efflux pump regulator mutations. Targeting antibiotic resistance in $B$. pseudomallei using molecular genotyping provides clinicians with a rapid tool to identify potential treatment failure in near real-time, enabling informed alteration of treatment during an infection and improved patient outcomes.

IMPORTANCE The melioidosis bacterium Burkholderia pseudomallei is intrinsically resistant to many antibiotics, limiting treatment options to a handful of drugs including meropenem, doxycycline and trimethoprim-sulfamethoxazole. Although rare, there have now been several documented melioidosis cases where resistance to these antibiotics has developed during an infection, leading to treatment failure and increased mortality rates. Interestingly, all strains resistant to these drugs exhibit increased efflux pump expression, representing a shared molecular signature that can be exploited for rapid diagnostic purposes. Here, we developed and validated a single-tube real-time QPCR assay to detect clinically relevant efflux pump upregulation in B. pseudomallei, an important first step towards high-level resistance. This triplex assay offers a drastically reduced turn-around-time compared to current methodology, enabling earlier detection of resistance emergence. Implementation of this new diagnostic will aid clinicians in the selection of appropriate therapy, thereby minimizing resistance development and treatment failure for this high-mortality disease.
Compiled April 17, 2018 This is a draft manuscript, pre-submission Address correspondence to Derek S. Sarovich, dsarovich@usc.edu.au.

E.P.P. and D.S.S. contributed equally to this work. 
Webb et al.

KEYWORDS: Burkholderia pseudomallei, antibiotic resistance, melioidosis, efflux pump, gene expression, qPCR

\section{INTRODUCTION}

The development of antibiotic resistance in Gram-negative bacteria has become a global crisis as declared by the World Health Organization (1) and Centers for Disease Control and Prevention (2). The Gram-negative bacterium B. pseudomallei, the causative agent of melioidosis, is one of the most intrinsically antibiotic resistant bacteria ( 3 , $4,5)$, due to its diverse array of chromosomally-encoded resistance mechanisms (6). This inherent resistance limits melioidosis treatment options to a small number of antibiotics. Resistance emergence to any of these drugs during treatment is thus of great concern given the limited number of alternative treatments.

Melioidosis is arguably one of the most neglected tropical diseases of our time, with gross underreporting of cases, particularly in emerging endemic regions (7). Recent modelling suggests that there are 165,000 melioidosis cases worldwide annually, of which 89,000 are fatal, rates that are similar to the much higher profile disease, measles (8). There is currently no vaccine towards B. pseudomallei, with treatment fully reliant on antibiotic administration, which is biphasic and lengthy. Treatment typically involves 1014 days of intravenous ceftazidime (CAZ), with meropenem (MEM) used in severe cases or when resistance develops towards CAZ (9). The eradication phase consists of at least three months of trimethoprim-sulfamethoxazole (SXT) or amoxicillin-clavulanate, with doxycycline (DOX) used in instances where patients develop impaired renal function, bone marrow suppression or skin reactions due to SXT intolerance (10). The emergence of resistance during treatment is fortunately rare, but has now been documented for almost all antibiotics used to treat melioidosis $(11,12,13,14,15)$, the exception being high-level (>12 $\mu \mathrm{g} / \mathrm{mL}$ ) MEM resistance. Alarmingly, cases of B. pseudomallei exhibiting decreased susceptibility to MEM have recently been identified in Australian patients (16), and are associated with prolonged blood culture positivity and poorer patient outcomes (17).

B. pseudomallei encodes for three clinically relevant resistance-nodulation-cell division (RND) efflux pumps: AmrAB-OprA (BSPL1802-BPSL1804), BpeAB-OprB (BPSL0814$B P S L 0816)$ and BpeEF-OprC (BPSS0292-BPSS0294), which give rise to resistance towards multiple antibiotic classes $(18,19,20)$. Each RND system consists of a membrane fusion protein (AmrA, BpeA, and BpeE, respectively), an RND transporter (AmrB, BpeB, and BpeF, respectively), an outer membrane protein (OprA, OprB, and OprC, respectively), and one or more regulators (AmrR [BPSL1805], BpeR [BPSL0812], and BpeT [BPSS0290] and BpeS [BPSL0731], respectively) (21). Overexpression of these efflux pumps can occur during the course of melioidosis treatment due to loss-of-function mutations in repressors or mutations leading to co-inducer independence of activators in their associated regulatory genes $(17,22,23,24)$. For instance, certain mutations affecting the TetR-type regulator gene, $a m r R$, cause decreased MEM susceptibilities in melioidosis patients with prolonged infections (17), and in combination with mutations in the SAM-dependent methyltransferase gene BPSL3085, can lead to clinically significant DOX resistance (23). The bpeT and bpeS genes encode two closely related LysR-type transcriptional regulators. Mutations that affect the carboxy-terminal co-inducer-binding domains of BpeT and BpeS, usually together with mutations in a tetrahydrofolate pathway-linked pterin reductase gene, ptr1/folM, have been associated with SXT resistance $(22,24)$. The bpe $R$ regulator is less well-characterized, although a mutation within this gene has been found in a clinical B. pseudomallei isolate that has intermediate 
Detection of efflux pump upregulation in B. pseudomallei

resistance to multiple drugs, including MEM (17). Importantly, all observed cases of MEM, DOX and SXT resistance have involved upregulation of one or more of these three RND efflux pumps $(17,25)$.

Despite the critical role that these RND efflux systems play in conferring acquired antibiotic resistance in $B$. pseudomallei, no methods are currently available to quickly and simultaneously detect their altered expression. A number of singleplex PCR assays have been published $(24,26,27,28)$; however, these assays are SYBR Green-based and thus cannot be multiplexed, making them more time-consuming and costly to perform. Additionally, SYBR Green-based assays are typically less robust and less specific than probe-based PCR assays due to the non-specific nature of the fluorogenic SYBR Green dye, which detects all double-stranded DNA molecules. Therefore, the aim of this study was to develop a robust and highly specific fluorogenic probe-based triplex quantitative real-time PCR (qPCR) assay to simultaneously detect the upregulation of the RND efflux pumps AmrAB-OprA, BpeAB-OprB and BpeEF-OprC in strains exhibiting increased minimum inhibitory concentrations (MICS) towards DOX, MEM and SXT. The triplex assay was first validated to determine the limits of quantitation (LoQ), detection (LoD), and linearity, and then tested against a panel of genetically characterized, antibiotic resistant $B$. pseudomallei isolates.

\section{RESULTS}

Limits of quantitation (LOQ) and detection (LOD), and linearity of the triplex qPCR assay. Following the design of probe-based assays targeting $a m r B, b p e B$ and bpeF, the lower LoQ and LoD was first calculated for each assay in the singleplex format, and subsequently in the triplex format. LoD was defined as the lowest analyte concentration at which detection is feasible, and LoQ was defined as the lowest concentration of analyte that can be determined with an acceptable level of precision and accuracy (29). Based on these definitions, the LoQ was determined as the lowest amount of DNA where $8 / 8$ replicates amplified with a CT standard deviation $(\sigma)$ of $<0.8$, and with good efficiency ( $R^{2}$ values $>0.98$ ), and LoD was defined as the concentration where at least $2 / 8$ replicates amplified, irrespective of $\sigma$ or efficiency. The lower LoQ in the singleplex format for all three assays was $\geq 4 \times 10^{-4} \mathrm{ng}$ ( $\geq 400 \mathrm{fg}$, 52 genomic equivalents (GEs)), and the LoD was $\geq 4 \times 10^{-6} \mathrm{ng}$ ( $\geq 4 \mathrm{fg}, 0.5 \mathrm{GEs}$ ), $\geq 4 \times 10^{-5} \mathrm{ng}$ ( $\geq 40 \mathrm{fg}, 5 \mathrm{GEs}$ ) and $\geq 4 \times 10^{-5}$ $\mathrm{ng}$ ( $\geq 40 \mathrm{fg}, 5 \mathrm{GEs}$ ) for $a m r B$, bpeF and bpeB, respectively. In the triplex format, for all targets, the LoQ was $\geq 4 \times 10^{-3} \mathrm{ng}$ ( $\geq 4 \mathrm{pg}, 515 \mathrm{GEs}$ ), and the LoD was $\geq 4 \times 10^{-5} \mathrm{ng}$ ( $\geq 40$ fg, 5 GEs) (Figure 1).

Next, the linearity of these assays was determined in the triplex and singleplex format. Efficiency (linearity) was measured by the rate at which a PCR amplicon is generated (30), where the maximum quantitative accuracy occurs in assays that function at or near $100 \%$ efficiency. In the triplex format, the standard curves for amrB, bpe $F$ and bpe $B$ all had an $\mathrm{R}^{2}$ value of $>0.95$ and showed good amplification efficiencies ( $90 \%$ for all three targets) (Figure S1). In singleplex format, the $R^{2}$ values were $>0.98$, with an efficiency of $>98 \%$ for all three assays (Figure S2).

The conserved genes mms and 23S rDNA are suitable housekeeping genes for normalized expression analysis in B. pseudomallei. To determine the performance of the conserved gene $m m s A$ as an expression normalization control for all clinical isogenic and Bp82 pairs, we compared normalized efflux pump expression against both $23 \mathrm{~S}$ and $\mathrm{mms} A$ in the eight Bp82 mutants. The normalized fold change of the triplex efflux assay for the eight Bp82 pairs was consistent when either $23 \mathrm{~S}$ or $m m s A$ was used for normalization (Table $\mathrm{S} 1$ ), suggesting that $m m s A$ expression, at least across the conditions that were tested in this study, is uniform. These results support 
bioRxiv preprint doi: https://doi org/10.1101/301960; this version posted April 17 2018. The copyright holder for this preprint (which was not certified by peer review) is the author/funder, who has granted bioRxiv a license to display the preprint in perpetuity. It is made available under aCC-BY-NC-ND 4.0 International license.

Webb et al.

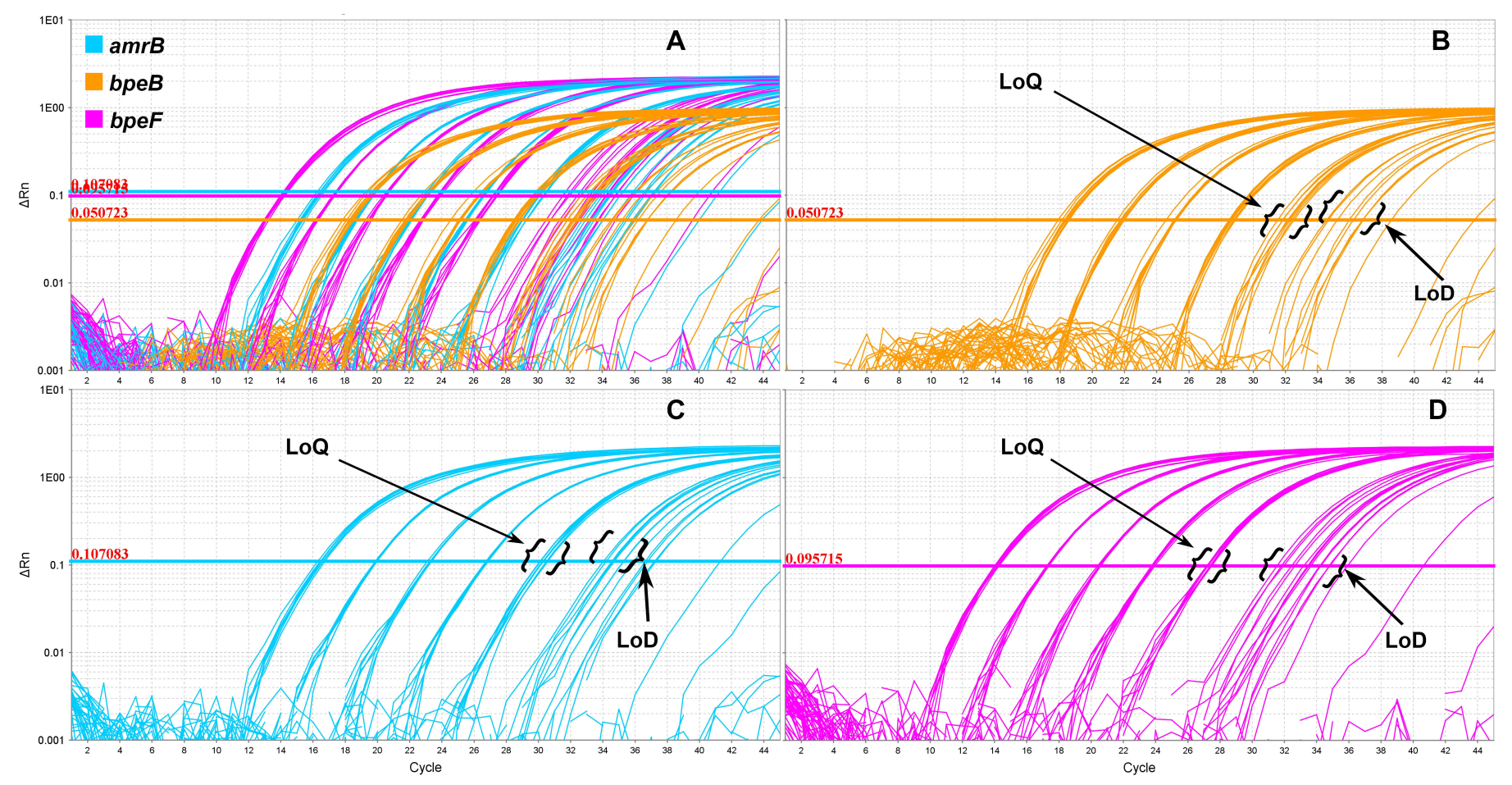

FIG 1 Quantitative PCR amplification plots of the Burkholderia pseudomallei resistance-nodulation-division efflux pump triplex assay. A) Amplification across eight replicates at DNA concentrations ranging from 40 to $4 \times 10-6 \mathrm{ng}$. B) Amplification plot of bpeB. C) Amplification plot of amrB. D) Amplification plot of bpeF. LoQ, limit of quantitation; LoD, limit of detection.

the use of $m m s A$ as a single-copy normalization control gene in B. pseudomallei.

Comparative genomic analysis identifies a novel amrR mutation in the latter P1048 isolate. SPANDx analysis of MSHR9766 and MSHR9872 was carried out to identify the genetic basis for decreased MEM susceptibility in the latter isolate. Two missense mutations were detected in MSHR9872: a mutation in AmrR ( $\left.\mathrm{AmrR}_{\mathrm{G} 50 \mathrm{E}}\right)$, and a mutation in the isoleucine tRNA synthetase gene, ileS (BPSL0906; $\|_{\text {leS }}{ }_{\mathrm{H} 505 \mathrm{G}}$ ), the product of which catalyzes the aminoacylation of Ile-tRNA. In addition, we detected a frameshift mutation in MSHR9872 within BPSS2161 (BPSS2161 A28fs), which encodes for a propanoate metabolism protein belonging to the MmgE-PrpD family. No other mutations (i.e. gene acquisition or loss, gene copy number variation) were identified between this pair.

Efflux pump upregulation in eight clinical strains with regulatory mutations. The triplex QPCR assay was tested on eight clinical B. pseudomallei isolates that encom-

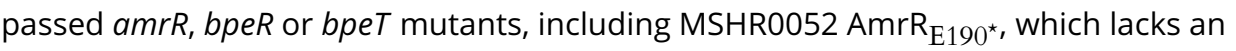
isogenic pair. None of the clinical strains tested in our study encoded bpes mutations. The triplex assay showed increased efflux expression in at least one of the RND efflux pumps in those isolates containing amrR, bpeR or bpeT regulatory mutations (Figure 2), consistent with their role in locally coordinating efflux pump expression. The latter strain from P215, MSHR0937, which encodes a mutation within the bpeAB-oprB regulator, bpeR (Bpe $\left.\mathrm{R}_{\mathrm{D} 176 \mathrm{~A}}\right)$, had a corresponding increase in the expression of bpe $B$ (15x; 3.8-fold; Figure 2A) compared with its WT isogenic pair, MSHR0664. Similarly, the latter strain isolated from Patient CF6, MSHR5654, which encodes a mutation within the bpeEF-oprC regulator, bpeT (BpeT T314fs $_{\text {) }}$, showed significant upregulation of bpeF (9.5x; 3.2-fold) when compared with its WT isogenic pair, MSHR5651 (Figure 2B).

Six latter strains, all of which exhibited decreased susceptibility towards MEM (3-8 


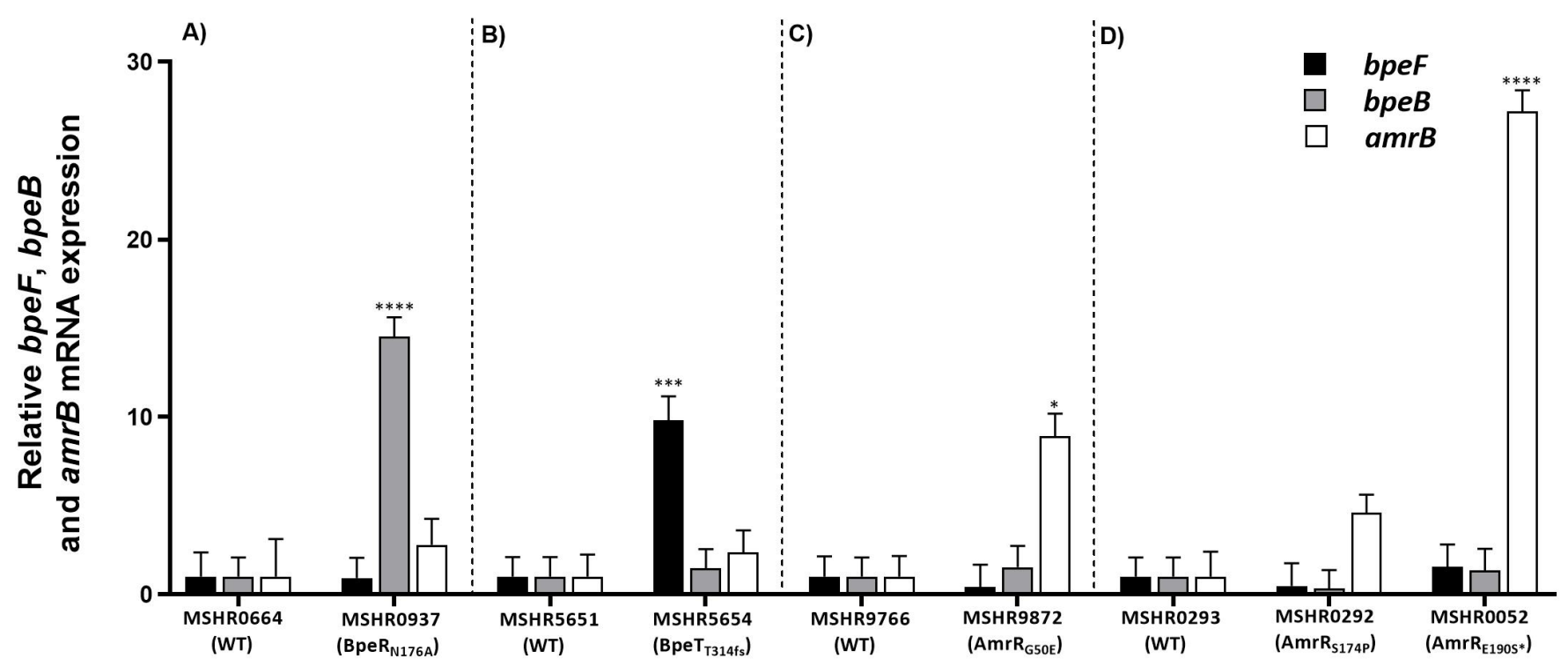

FIG 2 AmrAB-OprA (amrB), BpeAB-OprB (bpeB) and BpeEF-OprC (bpeF) expression in five melioidosis cases harboring B. pseudomallei isolates that encode efflux pump regulator mutations. As an isogenic WT B. pseudomallei pair is lacking for MSHR0052, MSHR0293 was used as the WT control for normalized efflux expression of this strain. For all other strains, the WT isogenic pair was used for normalization. Efflux pump expression was normalized against the conserved gene, $m m s A$ (31). Error bars denote standard deviation between biological replicates, which were all performed in technical duplicates. The y axis represents the relative times $(x)$ change in amrB, bpeB and bpeF expression. Statistical analysis was done by two-way ANOVA and Tukey's multiple-comparison test. ****, $p<0.0001$; ${ }^{* *}, p<0.001 ;{ }^{* *} p<0.01 ; *, p<0.05$.

$\mu \mathrm{g} / \mathrm{mL}$; Table 1), encoded mutations within the amrAB-oprA regulator, amrR. When these six strains were tested with the triplex qPCR assay, only three (MSHR9872 $\mathrm{AmrR}_{\mathrm{G50E}}, \mathrm{MSHR0292} \mathrm{AmrR}_{\mathrm{S} 174 \mathrm{P}}$ and MSHR0052 $\left.\mathrm{AmrR}_{\mathrm{E} 190^{*}}\right)$ showed a significant increase in $a m r B$ expression, which ranged from $5 \times$ (2.3-fold; MSHR0292 AmrR $\mathrm{A}_{\mathrm{S} 174 \mathrm{P}}$ ) to 27x (4.7-fold; MSHR0052 AmrR ${\mathrm{E} 190^{\star}}$ ) (Figures 2C and 2D). The remaining three $a m r R$ mutant strains, MSHR7929 AmrR E30D $_{1}$ MSHR4083 AmrR $\triangle$ A153-D156 and MSHR6755 $\mathrm{AmrR}_{\triangle \mathrm{V} 60-\mathrm{C} 63}$, showed no significant increase in $\mathrm{amr} B$ expression. Importantly, the amplification of the triplex assay for all the isogenic strains was within the LoQ of the triplex assay, ruling this factor out as a cause for the lack of differential expression.

Induction of AmrAB-OprA in B. pseudomallei amrR mutants exhibiting decreased MEM susceptibilities. To better understand the lack of differential expression of amrAB-oprA in some strains with altered $a m r R$, the triplex qPCR assay was tested on RNA extracted from MSHR4083, MSHR6755 and MSHR6755 $\triangle$ amrR grown in the presence of a sub-inhibitory $(0.25 \mu \mathrm{g} / \mathrm{mL})$ concentration of MEM. For MSHR6755 $\mathrm{AmrR}_{\triangle \mathrm{V} 60-\mathrm{C} 63}, a m r B$ was dramatically upregulated (24x; 4.6 -fold) in the presence of MEM (Figure $3 B$ ). Additionally, the triplex assay revealed a subtle (2x; 0.9 -fold) increase in the expression of bpeB and bpeF in this strain when grown in the presence of MEM (Figure 3B). In MSHR6755 $\triangle a m r R$, MEM induction also resulted in 12x (3.5-fold) $a m r B$ upregulation (Figure 3B). Similarly, MEM induction led to $21 \times$ (4.2-fold) upregulation

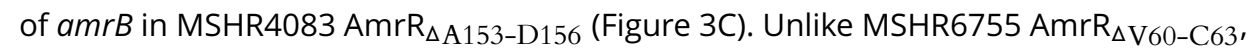
MEM did not induce bpeB and bpeF upregulation in MSHR6755 $\triangle a m r R$ or MSHR4083 $\mathrm{AmrR}_{\triangle \mathrm{A} 153-\mathrm{D} 156}$

Increased efflux expression in laboratory-generated efflux regulator mutants. The triplex qPCR assay was tested on eight laboratory-generated Bp82 mutants con- 
bioRxiv preprint doi: https://doi.org/10.1101/301960; this version posted April 17, 2018. The copyright holder for this preprint (which was not certified by peer review) is the author/funder, who has granted bioRxiv a license to display the preprint in perpetuity. It is made available under aCC-BY-NC-ND 4.0 International license.

Webb et al.

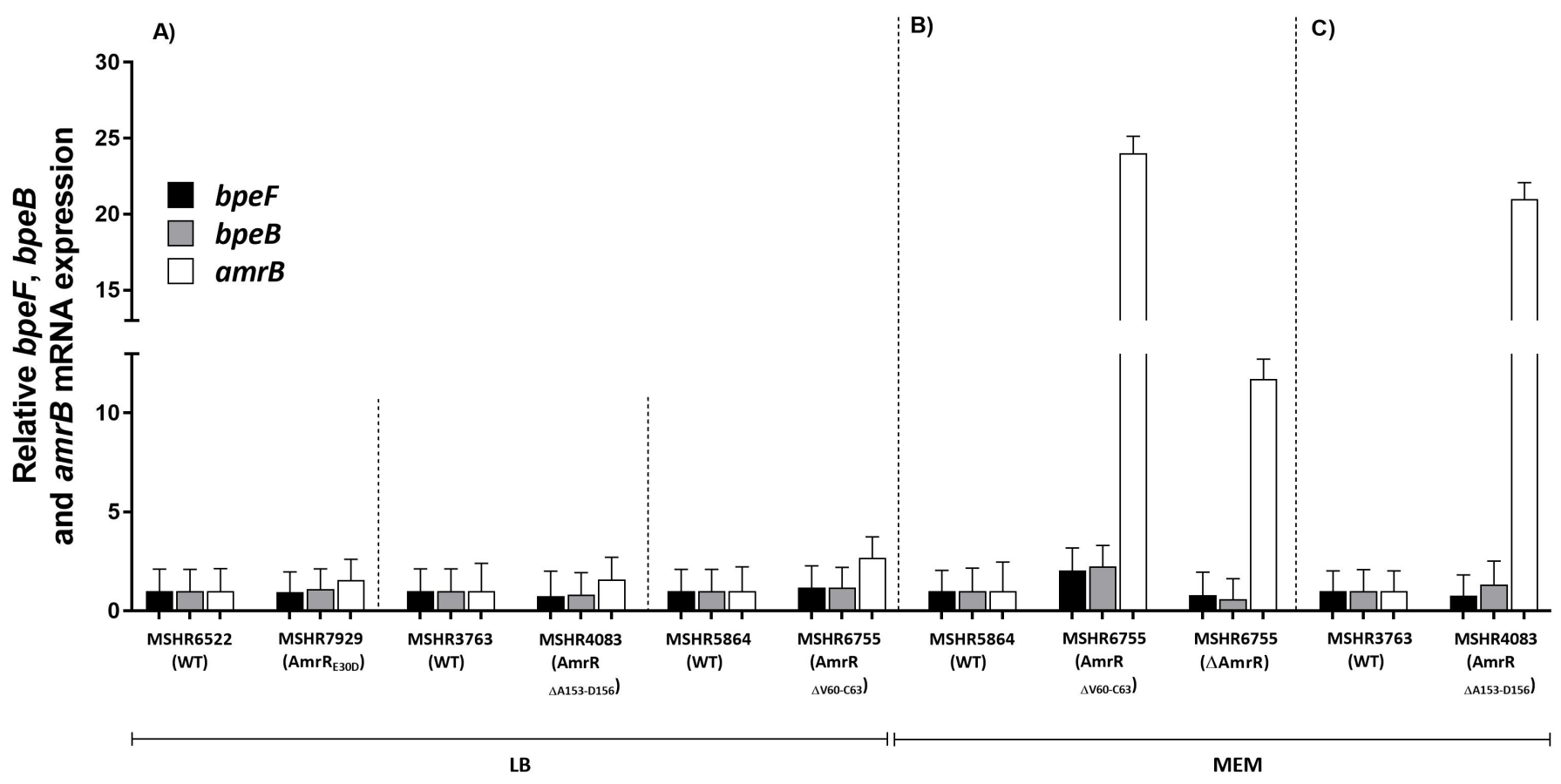

FIG 3 AmrAB-OprA (amrB), BpeAB-OprB (bpeB) and BpeEF-OprC (bpeF) expression in Burkholderia pseudomallei, with the latter isolate of each pair containing amrR point mutations or deletions that confer decreased meropenem (MEM) susceptibility. A) Three isogenic pairs grown in Luria-Bertani (LB) broth; B) isogenic pair MSHR5864 and MSHR6755 (and AmrR knockout MSHR6755 $\triangle a m r R$ ) grown in LB plus MEM; C) isogenic pair MSHR3763 and MSHR4083 grown in LB broth plus MEM. Efflux pump expression was normalized against the conserved gene, $m m s A$ (31). Error bars denote standard deviation between biological duplicates, which were all performed in technical duplicates. The $y$ axis represents the relative times $(x)$ change in amr $B, b p e B$ and $b p e F$ expression. Statistical analysis was done by two-way ANOVA and Tukey's multiple-comparison test, a statistically significant $p$ value was not identified between pairs. 


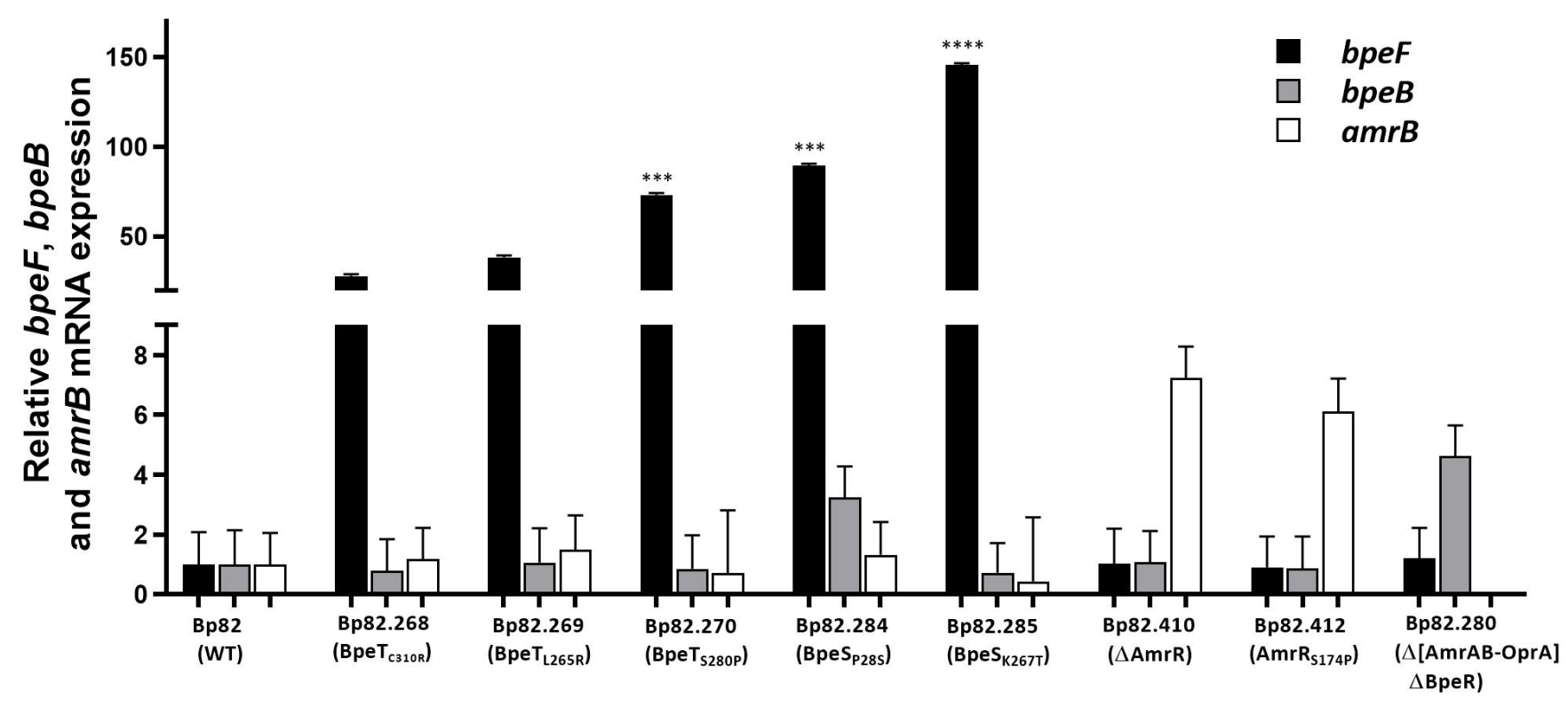

FIG 4 AmrAB-OprA (amrB), BpeAB-OprB (bpeB) and BpeEF-OprC (bpeF) expression in eight laboratory-generated Burkholderia pseudomallei Bp82-derived mutants containing regulator mutations relative to WT Bp82. Error bars denote standard deviation between biological triplicates, which were all performed in technical triplicates. Efflux pump expression was normalized against the conserved gene, $m m s A(43)$. The y axis represents the relative times (x) change in amr $B$, bpeB and bpeF expression. Statistical analysis was done by two-way ANOVA and Tukey's multiple-comparison test. $* \star \star \star, p<0.0001 ; * \star *, p<0.001$.

taining mutations affecting the bpeT, bpeS or amrR efflux pump regulators (Figure 4). The three bpeT missense mutants, Bp82.268 BpeT $\mathrm{C}_{310 \mathrm{R}}, \mathrm{Bp} 82.269 \mathrm{BpeT}_{\mathrm{L} 265 \mathrm{R}}$

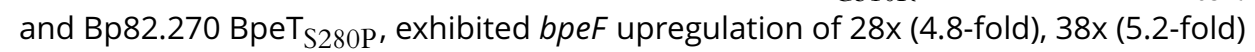
and $73 \times$ (6.1-fold), respectively, but no significant upregulation of the other two efflux pumps. The two bpes missense mutants, Bp82.284 BpeS $\mathrm{P} 29 \mathrm{~S}_{\text {and }}$ Bp82.285 BpeS $\mathrm{K} 267 \mathrm{~T}_{\text {, }}$ exhibited upregulation of bpeF of $89 x$ (6.5-fold) and 145x (7.2-fold), respectively. In Bp82.284 BpeS 299 , bpeB was also moderately upregulated ( $3 x_{;} ; 1.5$-fold). Upregulation of $a m r B$ was confirmed in the two amrR mutants Bp82.412 AmrR $_{\mathrm{S} 174 \mathrm{P}}(6 \mathrm{x} ; 2.6-$ fold) and Bp82.410 $\triangle a m r R$ (7x; 2.8-fold), with no demonstrable differential expression of bpeB and bpeF. Lastly, in Bp82.280 $\Delta$ (amrAB-oprA) $\triangle b p e R$, the triplex qPCR assay showed no $a m r B$ amplification due to deletion of this locus, but $5 \times$ (2.3-fold) upregulation of bpe $B$ due to deletion of bpeR. In all instances where amplicons were produced, triplex assay amplification for the isogenic Bp82 mutants was within the LoQ of the triplex assay.

\section{DISCUSSION}

Despite dramatic improvements in survival rates over the past 30 years, melioidosis continues to have a stubbornly high fatality rate, with between 10 and $40 \%$ of treated cases in the hyperendemic regions of northern Australia and Northeast Thailand, respectively, resulting in death (32). It is becoming clearer that at least some of these cases can be linked to the development of previously unrecognized resistance or decreased susceptibility towards clinically relevant antibiotics $(17,22,33,34)$. Of most concern, antibiotic resistance in B. pseudomallei has now been documented towards almost all antibiotics (17). Identification of an increasing number of novel acquired antibiotic resistance mechanisms in clinical B. pseudomallei strains, together with the 
Webb et al.

broadening global presence of melioidosis-endemic regions, has increased the urgency for methods that can provide rapid detection of emerging antibiotic resistance.

In B. pseudomallei, antibiotic resistance determination currently relies on culturebased diagnostics including inhibition zone measurement (e.g. Etests) or serial dilutions of the antibiotic in culture media (e.g. broth or agar microdilutions) (35). Despite culture-based methods being accurate, they have lengthy turn-around-times, and do not determine the genetic basis of resistance, both of which are important considerations when determining optimal therapeutic strategies. Nucleic-acid based detection methods such as real-time PCR have the potential to circumvent many shortcomings of traditional culture-based methods, offering more rapid turn-around times and determination of the underlying resistance mechanism, which is essential for identifying potential cross-resistance mechanisms $(33,36)$ or stepwise progression towards highlevel resistance development $(23,37,38)$. Information generated from such assays has the potential to inform optimal treatment strategies for melioidosis patients in near real-time and reduce the number of cases where undiagnosed antibiotic resistance emerges during treatment (39).

RND efflux pump upregulation is common in B. pseudomallei strains that exhibit decreased susceptibility towards clinically relevant antibiotics. This mechanism can either by itself, or in concert with additional mutations, cause resistance towards DOX, SXT, and MEM $(17,22,23,24)$, three invaluable antibiotics in melioidosis treatment regimens. Therefore, the main goal of this study was to design, optimize and validate a triplex qPCR probe-based assay targeting the AmrAB-OprA, BpeAB-OprB, and BpeEFOprC RND efflux pumps in B. pseudomallei (40). Several nucleic acid-based assays have been designed for the identification of antibiotic resistance in $B$. pseudomallei $(26,33,34,41,42)$; however, all are in singleplex format, making them unattractive for characterizing more than a handful of antibiotic resistance-conferring mutations. In contrast, probe-based PCR methods can simultaneously identify multiple targets in a single assay while being efficient, affordable, sensitive and specific.

Given the large number of mutations that can cause RND efflux pump upregulation $(17,22,23,28,43)$, we opted for a multiplex probe-based assay format that identifies increased gene expression in favor of designing individual assays targeting each regulatory mutation, the latter of which would be both impractical (due to the large number of assays required) and prone to false negatives (due to the high likelihood of missing novel variants). In addition, detecting RNA expression ensures that efflux pump upregulation is identified without requiring a thorough understanding of the underlying regulatory networks. We first optimized the three highly specific efflux pump qPCR assays in both the singleplex and triplex formats, with all assays demonstrating high robustness and sensitivity levels, similar to the highly robust $B$. pseudomallei mmsA probe-based assay (31). Following optimization, we tested the triplex qPCR assay on 16 clinical and laboratory-generated $B$. pseudomallei mutants that had alterations in amrR, bpeR, bpeT or bpeS and which exhibited resistance towards DOX or SXT or had decreased MEM susceptibilities. We demonstrated that the triplex qPCR assay accurately quantified upregulation of efflux in all 16 regulatory mutants. Our findings confirm the value of the triplex qPCR assay for the detection of RND efflux pump upregulation.

The mechanisms conferring DOX and SXT resistance, and decreased MEM susceptibility, have recently been unraveled in B. pseudomallei. Importantly, all resistant strains analyzed to date have upregulated efflux pump expression $(17,22,23,24)$. Our study confirmed that upregulation of $a m r B, b p e B$ or bpeF was always associated with decreased MEM susceptibility. Thus, in a clinical setting, the triplex assay can be used 
Detection of efflux pump upregulation in B. pseudomallei

for the accurate detection of B. pseudomallei isolates exhibiting decreased MEM susceptibility without the need for MIC determination. In contrast, additional mutations outside of efflux pump regulatory regions, which affect either the methyltransferase, $B P S L 3085$, or the tetrahydrofolate synthesis pathway-linked enzyme pterin reductase, folM/ptr1, are required for the shift towards high-level DOX and SXT resistance $(23,24)$. The two-step nature of these mechanisms means that the triplex qPCR assay alone cannot be used to definitively determine if a strain is resistant to DOX or SXT. However, the assay can be used to rule out antibiotic resistance, as a strain with little or no efflux activity is highly likely to be sensitive to these drugs. Importantly, the ability to rapidly screen isolates and detect precursor mutations to clinically relevant DOX and SXT resistance provides a unique opportunity for health practitioners to make informed decisions regarding the suitability of current treatment, and to be aware of the risk of future resistance emergence towards these antibiotics. If increased efflux pump activity is detected in any B. pseudomallei strain, we strongly recommend that isolates be subjected to further MIC testing and/or the patient's treatment regimen altered to avoid selecting for resistance emergence and subsequent treatment failure.

It has been previously shown that MEM induces the expression of AmrAB-OprA in amrR-mutated B. pseudomallei strains, leading to decreased MEM susceptibility (17). In the current study, the triplex qPCR assay confirmed that some, but not all, amrR mutants required MEM for the induction of AmrAB-OprA upregulation. In contrast, the two tested WT strains, MSHR5864 and MSHR3763, did not show an increase in the expression of $a m r B$ in the presence of MEM. Efflux pump expression was inducible in strains encoding in-frame, four-residue amrR deletions (MSHR4083 $\mathrm{AmrR}_{\triangle \mathrm{A} 153-\mathrm{D} 156}$ and MSHR6755 $\mathrm{AmrR}_{\triangle \mathrm{V} 60-\mathrm{C} 63}$ ), and when amrR was completely lost (MSHR6755 $\triangle a m r R$ ); however, induction was not required for two strains encoding either a point mutation (MSHR0292 AmrR S174P) $_{\text {) }}$ or a premature stop codon (MSHR0052 $\mathrm{AmrR}_{\mathrm{E} 190^{\star}}$ ). The reason for this difference is not yet fully understood. One possibility is that RND efflux pump regulatory genes are capable of binding to substrates of the efflux systems that they regulate, and in doing so, dissociate from their promoter regions, leading to efflux pump upregulation $(44,45)$. This hypothesis may explain the molecular mechanism in the strains that encode the in-frame amrR deletions. However, this concept cannot be applied to the strain lacking $a m r R$, as the regulator is not present, which would lead to efflux pump expression even in the absence of MEM. Although not examined in this study, it is possible that a second, as-yet-undiscovered regulator may also be involved in amrAB-oprA regulation. Alternately, certain mutation types or methylation patterns may reverse the function of AmrR, resulting in activation rather than repression of the operon. Further work using methods such as transcriptomics (RNA-seq) or bisulfite sequencing (methyl-seq) would be needed to confirm this hypothesis. Based on our results, we recommend that a sub-inhibitory concentration of MEM (i.e. $0.25 \mu \mathrm{g} / \mathrm{mL}$ ) is sufficient to induce RND efflux pump upregulation in strains with decreased MEM susceptibility, without impacting RND efflux pump expression in WT strains. Although not investigated here, MEM may not be required for induction if RNA is extracted directly from clinical samples due to the preservation of native expression/methylation profiles, or even the presence of MEM in vivo upon sample collection.

There are some recognized limitations to our study. First, there are greater difficulties associated with handling RNA compared with DNA due to the high lability of bacterial mRNA if not protected appropriately (46). This requirement limits the application of our assay to freshly collected specimens, appropriately preserved material, or viable cultures. Second, we only assessed the use of our triplex assay on RNA extracted 
Webb et al.

from subcultured isolates, and our TRIzol-based RNA extraction method is relatively slow $\sim 4 \mathrm{~h}$ from pelleted culture to purified and quality-controlled RNA). These factors pose a significant barrier to the rapid diagnosis of RND efflux pump upregulation. An assessment of rapid RNA extraction methods from bacterial cultures, or ideally, directly from clinical specimens $(47,48)$, is needed to achieve same-day identification of strains with increased RND efflux expression. Finally, the complex nature of antibiotic resistance mechanisms may act as a barrier to the uptake of molecular antibiotic resistance assays in clinical laboratories due to an unfamiliarity with molecular techniques or difficulties in communicating results among healthcare professionals. The latter issue can be overcome with the availability of report templates, such as the Mycobacterium tuberculosis template for antibiotic resistance determination in tuberculosis patients based on WGS data, which communicates molecular results from antibiotic resistance strains to health practitioners in a clear and unambiguous manner, and without the need for specialized molecular knowledge (49).

\section{CONCLUSIONS}

B. pseudomallei is an important mammalian pathogen that is endemic in most tropical and many subtropical regions. As new endemic regions continue to be unveiled, and as better recognition of this neglected pathogen filters through to the clinical setting, the number of melioidosis cases reported globally is expected to increase dramatically. Due to the high mortality rate of this disease even with antibiotic treatment, patient management should include regular screening of clinical isolates for the emerging development of antibiotic resistance to ensure optimal treatment strategies are being implemented, and to minimize the possibility of unintended treatment failure. With this goal in mind, we developed and optimized a triplex qPCR assay that simultaneously detects the expression of AmrAB-OprA, BpeAB-OprA and BpeEF-OprC in B. pseudomallei isolates. The triplex assay was tested on a panel of genetically characterized $B$. pseudomallei isolates of both clinical and laboratory origin, with known mutations leading to SXT or DOX resistance, or decreased MEM susceptibilities. In all B. pseudomallei strains that contained regulatory mutations, upregulation of at least one efflux pump was accurately detected. Rapid and facile detection of efflux upregulation is a crucial component of detecting emerging drug resistance in B. pseudomallei, and will aid in prompt and effective administration of individualized treatment regimens for melioidosis patients.

\section{MATERIALS AND METHODS}

Ethics statement. This study was approved by the Human Research Ethics Committee of the Northern Territory Department of Health and the Menzies School of Health Research (HREC 02/38).

Melioidosis patients and corresponding B. pseudomallei clinical isolates. Eight Australian melioidosis cases were examined in this study. Seven cases have previously been described, including one (Pre-DPMS 89) that lacks a wild-type (WT) isogenic pair. Prior comparative genomic analysis and functional characterization showed that all non-WT strains from these patients encode RND efflux pump regulatory mutations that cause increased MICs (either resistance or decreased susceptibility) towards SXT, $\operatorname{DOX}$ or MEM $(16,17,22,23)$.

During this study, an adult patient, P1048, presented with acute pneumonia to Royal Darwin Hospital, Northern Territory, Australia. Initial treatment involved MEM for four weeks, followed by six weeks of CAZ and then a three-month eradication course of DOX due to SXT intolerance (neutropenia). MSHR9766 was cultured from 
Detection of efflux pump upregulation in B. pseudomallei

sputum upon initial admission, and MSHR9872 was cultured from sputum during the final week of MEM treatment. P1048 reverted to being culture-negative after two months of treatment, and has since recovered from their infection. MIC testing showed that MSHR9766 was sensitive to MEM $(1 \mu \mathrm{g} / \mathrm{mL})$, whereas MSHR9872 had decreased susceptibility towards MEM $(7 \mu \mathrm{g} / \mathrm{mL})$, although both isolates were sensitive towards the other clinically relevant antibiotics (DOX, SXT and CAZ). Due to the development of an elevated MEM MIC in a latter isolate from this patient, we included this patient in our study.

The strain details for these eight cases, including regulatory mutations and MIC data, are shown in Table 1. Additionally, an $a m r R$ knockout of the final isolate retrieved from Patient 726 (MSHR6755 $\Delta a m r R$ ), which was previously created via allelic exchange (17), was included in the present study to determine the effect of regulator loss on efflux expression profiles.

B. pseudomallei growth conditions and MIC determination. All clinical strains were grown on Luria-Bertani (LB) agar or in LB broth (Oxoid, Thebarton, SA, Australia) at $37^{\circ} \mathrm{C}$ for $24 \mathrm{~h}$ unless otherwise stated. For the select agent-excluded strain Bp82 (50) and its derivatives, media were supplemented with $80 \mu \mathrm{g} / \mathrm{mL}$ adenine (Sigma, St. Louis, MO, USA), and for MIC testing of Bp82-derived strains, Mueller-Hinton (MH) agar or broth was supplemented with $40 \mu \mathrm{g} / \mathrm{mL}$ adenine. Escherichia coli strains DH5 $\alpha$ and $\mathrm{RHO} 3$ were used for plasmid DNA manipulation or mobilization, respectively, and were grown according to previously published methods $(51,52)$. Antimicrobial susceptibility testing was performed using Etests according to the manufacturer's instructions (bioMérieux, Baulkham Hills, NSW, Australia). Resistance cut-offs were based on the Clinical and Laboratory Standards Institute (CLSI) guidelines, as follows: CAZ $\mathrm{S} \leq 8, \mathrm{I}=16$ and $\mathrm{R} \geq 32 \mu \mathrm{g} / \mathrm{mL} ; \mathrm{DOX}: \mathrm{S} \leq 4, \mathrm{I}=8$ and $\mathrm{R} \geq 16 \mu \mathrm{g} / \mathrm{mL}$; and $\mathrm{SXT}: \mathrm{S} \leq 2 / 38$ and $\mathrm{R} \geq 4 / 76 \mu \mathrm{g} / \mathrm{mL}$ (53). The CLSI guidelines do not list MIC values for $B$. pseudomallei towards MEM; thus we categorized decreased MEM susceptibility as MICs $\geq 3 \mu \mathrm{g} / \mathrm{mL}$ based on prior studies $(17,54,55)$. All experiments with clinical $B$. pseudomallei isolates were performed in a physical containment level 3 (biosafety level 3) facility according to local regulations, whereas experiments involving strain Bp82 and its derivatives were conducted in the physical containment level 2 laboratory, as Bp82 is excluded from select agent regulations due to an attenuated virulence phenotype conferred by a $\Delta$ purM mutation (www.selectagents.gov/SelectAgentsandToxinsExclusions.html).

B. pseudomallei Bp82 efflux pump regulator mutants. Eight $B$. pseudomallei Bp82 strains with efflux pump regulatory mutations were included, six of which have been previously created (24): Bp82.268 BpeT $\mathrm{C} 310 \mathrm{R}_{1}, \mathrm{Bp} 82.269 \mathrm{BpeT}_{\mathrm{L} 265 \mathrm{R}}, \mathrm{Bp} 82.270$ BpeT $_{\mathrm{S} 280 \mathrm{P}}, \mathrm{Bp} 82.284 \mathrm{BpeS}_{\mathrm{P} 29 \mathrm{~S}}, \mathrm{Bp82.285} \mathrm{BpeS}_{\mathrm{K} 267 \mathrm{~T}}$ and Bp82.280 $\Delta(a m r A B-o p r A)$ $\triangle b p e R$. These Bp82 mutants, with the exception of Bp82.280 $\Delta$ (amrAB-oprA) $\triangle b p e R$, were created via site-directed mutagenesis and knockouts to investigate the mechanisms of SXT resistance in B. pseudomallei using previously described methods (24). Bp82.280 $\triangle$ (amrAB-oprA) $\triangle b p e R$ was derived from a previously created strain (Bp82.27) as previously described $(27,56)$ leaving a Flp recombinase target (FRT) scar in the bpe $R$ gene. MIC data for the Bp82 mutants are detailed in Table 1.

Construction of two novel Bp82 amrR mutants. We have recently shown that a $\mathrm{T}_{520 \mathrm{C}}$ point mutation in $\operatorname{amrR}\left(\mathrm{AmrR}_{\mathrm{S} 174 \mathrm{P}}\right.$ ) in an Australian clinical B. pseudomallei isolate plays an important role in increasing the MIC towards DOX (1 to $16 \mu \mathrm{g} / \mathrm{mL}$ ) (23). Two laboratory-generated Bp82 amrR mutants (Bp82.410 $\triangle a m r R$ and Bp82.412 $A m r R_{S 174 P}$ ) were therefore created in this study to better understand the effect of amrR mutations on amrAB-oprA expression. Deletion of $a m r R$ was achieved using the pEXKm5-based allelic replacement system (51) (Table 2). Briefly, the US and DS region 
bioRxiv preprint doi: https://doi.org/10.1101/301960; this version posted April 17, 2018. The copyright holder for this preprint (which was not certified by peer review) is the author/funder, who has granted bioRxiv a license to display the preprint in perpetuity. It is made available under aCC-BY-NC-ND 4.0 International license.

Webb et al.

TABLE 1 Burkholderia pseudomallei clinical isolates and laboratory-generated mutants used in this study. Mutations in resistancenodulation-division efflux regulatory genes are indicated. Minimum inhibitory concentrations (MICs; $\mu \mathrm{g} / \mathrm{mL}$ ) for wild-type and mutant strains are shown for each antibiotic. MICs above the intermediate (i) or resistant ( $r$ ) threshold determined for $B$. pseudomallei are indicated.

\begin{tabular}{|c|c|c|c|c|c|c|c|c|c|}
\hline Patient & Strain & Regulatory mutation & DOX & TMP & SMX & SXT & CAZ & MEM $^{a}$ & Study \\
\hline \multicolumn{10}{|c|}{ Clinical strains } \\
\hline Pre-DPMS 89 & MSHR0052 & $\mathrm{AmrR}_{\mathrm{E} 190^{b}}$ & $48(r)$ & ND & ND & ND & ND & 8 (i) & (17) \\
\hline \multirow[t]{2}{*}{ Non-DPMS 00} & MSHR0293 & WT & 1 & ND & ND & 0.38 & 1 & 0.5 & (23) \\
\hline & MSHR0292 & $\mathrm{AmrR}_{\mathrm{S} 174 \mathrm{P}}$ & $16(r)$ & ND & ND & 0.50 & 1 & 2 & \\
\hline \multirow[t]{2}{*}{ CF6 } & MSHR5651 & WT & 0.38 & ND & ND & 0.75 & 1.5 & 0.5 & (22) \\
\hline & MSHR5654 & $\mathrm{BpeT}_{\mathrm{T} 314 \mathrm{fs}}$ & 3 & ND & ND & $>32(r)$ & $>256(r)$ & 2 & \\
\hline \multirow[t]{2}{*}{ P215 } & MSHR0664 & WT & ND & ND & ND & 2 & 0.75 & 1.5 & (17) \\
\hline & MSHR0937 & $\mathrm{BpeR}_{\mathrm{N} 176 \mathrm{~A}}$ & 4 & ND & ND & 2 & 8 & 6 (i) & \\
\hline \multirow[t]{2}{*}{ P1048 } & MSHR9766 & WT & ND & ND & ND & ND & ND & 0.5 & This study \\
\hline & MSHR9872 & $\mathrm{AmrR}_{\mathrm{G} 50 \mathrm{E}}$ & ND & ND & ND & ND & ND & 6 (i) & \\
\hline \multirow[t]{2}{*}{ P797 } & MSHR6522 & WT & 1 & ND & ND & 1.5 & 1.5 & 0.5 & (17) \\
\hline & MSHR7929 & $\mathrm{AmrR}_{\mathrm{E} 30 \mathrm{D}}$ & 2 & ND & ND & $4(r)$ & 1.5 & 4 & \\
\hline \multirow[t]{2}{*}{ P608 } & MSHR3763 & WT & 0.75 & ND & ND & 3 (i) & 2 & 0.75 & (17) \\
\hline & MSHR4083 & $\mathrm{AmrR}_{\Delta \mathrm{A} 153-\mathrm{D} 156}$ & 1 & ND & ND & $24(r)$ & 2 & 6 (i) & \\
\hline \multirow[t]{3}{*}{ P726 } & MSHR5864 & WT & 1 & ND & ND & 1.5 & 1.5 & 0.75 & (17) \\
\hline & MSHR6755 & $\mathrm{AmrR}_{\Delta \mathrm{V} 60-\mathrm{C} 63}$ & 1.5 & ND & ND & 0.75 & 1.5 & 3 & \\
\hline & MSHR6755 & $\Delta a m r R$ & ND & ND & ND & ND & ND & 3 & \\
\hline \multicolumn{10}{|l|}{ Bp82 mutants } \\
\hline $\mathrm{N} / \mathrm{A}$ & Bp82 & WT & 0.5 & 0.75 & 4 & 0.094 & 2 & ND & (50) \\
\hline N/A & Bp82.268 & $\mathrm{BpeT}_{\mathrm{C} 310 \mathrm{R}}$ & 2 & 4 & 8 & 0.38 & 2 & ND & (24) \\
\hline N/A & Bp82.269 & $\mathrm{BpeT}_{\mathrm{L} 265 \mathrm{R}}$ & 1 & 4 & 8 & 0.38 & 2 & ND & (24) \\
\hline N/A & Bp82.270 & BpeT $_{\text {S280P }}$ & 8 & $>32$ & ND & ND & 2 & ND & (24) \\
\hline N/A & Bp82.284 & $\mathrm{BpeS}_{\mathrm{P} 29 \mathrm{~S}}$ & 2 & $>32$ & ND & ND & $2-4$ & ND & (24) \\
\hline N/A & Bp82.285 & $\mathrm{BpeS}_{\mathrm{K} 267 \mathrm{~T}}$ & 8 & $>32$ & ND & ND & $1-2$ & ND & (24) \\
\hline N/A & Bp82.280 & $\triangle$ AmrAB-OprA $\triangle$ BpeR & 1 & 0.75 & ND & ND & 2 & ND & $(24,27)$ \\
\hline N/A & Bp82.410 & $\Delta \mathrm{AmrR}$ & 4 & 0.75 & ND & ND & 2 & ND & This study \\
\hline N/A & Bp82.412 & $\mathrm{AmrR}_{\mathrm{S} 174 \mathrm{P}}$ & 4 & 0.75 & ND & ND & 2 & ND & This study \\
\hline
\end{tabular}


bioRxiv preprint doi: https://doi.org/10.1101/301960; this version posted April 17, 2018. The copyright holder for this preprint (which was not certified by peer review) is the author/funder, who has granted bioRxiv a license to display the preprint in perpetuity. It is made available under aCC-BY-NC-ND 4.0 International license.

Detection of efflux pump upregulation in B. pseudomallei

TABLE 2 Plasmids used in the study.

\begin{tabular}{lll}
\hline Plasmid & Description & Source \\
\hline pEXKm5 & $\mathrm{Km}^{r} ;$ allelic-exchange plasmid & $(51)$ \\
pEXKm5-US-DS-amrR & $\mathrm{Km}^{r} ;$ pEXKm5 with US-DS- $a m r R$ & This study \\
pEXKm5-US-DS-amrRT520C & $\mathrm{Km}^{r} ;$ pEXKm5 with US-DS- $a m r R_{\mathrm{T} 520 \mathrm{C}}{ }^{2}$ & This study \\
pGEM-T Easy & $\mathrm{Ap}^{r} ;$ PCR amplicon cloning vector & Promega \\
pGEM-T-US-DS-WT- $a m r R$ & $\mathrm{Ap}^{r} ;$ pGEM-T Easy with US-DS-WT- $a m r R$ PCR fragment from Bp82 & This study \\
pGEM-T-US-DS- $a m r R_{\mathrm{T} 520 \mathrm{C}}$ & $\mathrm{Ap}^{r} ;$ pGEM-T Easy with US-DS- $a m r R_{\mathrm{T} 520 \mathrm{C}}$ created via site directed mutagenesis & This study \\
\hline
\end{tabular}

Abbreviations: US, upstream; DS, downstream; Kmr, kanamycin resistant; Apr, ampicillin resistant; WT, wild-type.

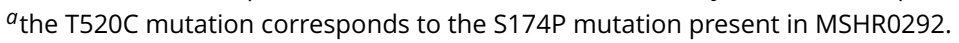

of $a m r R$ was amplified by PCR (using Bp82 genomic DNA as template) using primers amrR_UP_F, amrR_UP_R, amrR_DN_F and amrR_DN_R (Table 3). The PCR fragments were purified and assembled into pEXKm5 to create pEXKm5-US-DS-amrR (Table 2), using NEBuilder High-Fidelity DNA Assembly system (New England Biolabs, Ipswich, MA, USA). pEXKm5-US-DS-amrR was subsequently conjugated into Bp82 using an $E$. coli RHO3 mobilizer strain (51). Finally, merodiploids were selected for and resolved. The resultant $\triangle a m r R$ strain was named Bp82.410. Loss of $a m r R$ was confirmed by PCR amplification using the primers amrR_Fullgene_F and amrR_Fullgene_R (Table 3), with the amplified size of WT amrR being 1,472bp compared with the amrR knockout at $800 \mathrm{bp}$. For construction of strain Bp82.412 expressing AmrR $\mathrm{S}_{\mathrm{S} 174 \mathrm{P}}$, the entire $a m r R$ gene including $400 \mathrm{bp}$ upstream sequence (US) and $400 \mathrm{bp}$ downstream sequence (DS) was first ligated into pGEM-T Easy (Promega, Madison, WI, USA) (Table 2) to create pGEM-T-US-DS-WT-amrR (Table 2) using primers amrR_Fullgene_F and amrR_Fullgene_R (Table 3). Site-directed mutagenesis was performed using pGEM-T-US-DS-WT-amrR together with the amrR mutagenic primers amrR_T520C_F and amrR_T520C_R (Table 3) to create pGEM-T-US-DS-amrRT520C (Table 2) following the manufacturer's instructions (QuikChange II Site-Directed Mutagenesis Kit, Agilent Technologies, Santa Clara, CA, USA). The US-DS-amrR ${ }_{\mathrm{T} 520 \mathrm{C}}$ fragment was subcloned into the EcoRI site of pExKm5 creating pEXKm5-US-DS-amrR ${ }_{\text {T520C }}$ (Table 2). Mutagenesis was confirmed by dideoxy nucleotide sequencing of the newly introduced $\mathrm{amrR}_{\mathrm{T} 520 \mathrm{C}}$. The mutagenic plasmid pEXKm5-US-DS-amrR ${ }_{T 520 C}$ was then introduced into Bp82.410 $\Delta a m r R$ as described using E. coli RHO3 (51), followed by allelic exchange. Merodiploids were selected for and resolved to create Bp82.412 (Bp82 AmrR $\left.{ }_{\mathrm{S} 174 \mathrm{P}}\right)$.

Genomic analyses. We have previously used a comparative genomics approach to identify genetic variants in seven cases $(16,17,22,23)$. This same approach was also employed to identify variants between isolates obtained from a new melioidosis case, P1048, which arose during the course of our study. Reference-based assembly of the initial P1048 isolate, MSHR9766, was performed with MGAP V1.0 (https://github.com/dsarov/MGAP-Microbial-Genome-Assembler-Pipeline), using the closed genome of Australian strain MSHR1153 (57) for scaffolding. The MSHR9766 assembly was error-corrected by self-read mapping to correct for a small number of single-nucleotide polymorphism (SNP) and insertion-deletion (indel) errors. The corrected assembly was subsequently used as a reference to identify genetic variants (SNPs, indels, copy number variants) between the P1048 strains, and to rule out gene loss in the latter strain. Read mapping was carried out using BWA (58), SAMTools (59), GATK (60) and SnpEff (61), which are wrapped in the SPANDx pipeline v3.2.1 (62). Default SPANDx settings were used, with the flag for indel detection (-i) enabled. A second analysis using the Thai clinical strain B. pseudomallei K96243 (63) as the reference 
bioRxiv preprint doi: https://doi.org/10.1101/301960; this version posted April 17, 2018. The copyright holder for this preprint (which was not certified by peer review) is the author/funder, who has granted bioRxiv a license to display the preprint in perpetuity. It is made available under aCC-BY-NC-ND 4.0 International license.

Webb et al.

TABLE 3 Primers and probes used for amrR knockout, site-directed mutagenesis and detection of efflux pump upregulation.

\begin{tabular}{ll}
\hline Primers/Probe & Sequence (5'-3') \\
\hline Deletion of $\boldsymbol{a m r \boldsymbol { R }}$ & \\
amrR_UP_F & CCTGTTATCCCTACCCGGGCTCCTGCCCTTCCTCGTAGG \\
amrR_UP_R & CAGTGCGCGTGGGGGGGGTGCTCCCTTA \\
amrR_DN_F & CACCCCCCCACGCGCACTGAACGCGCA \\
amrR_DN_R & GGGATAACAGGGTAATCCCGGCGGGCGGAAGAAACGGAAAC \\
Amplification of $\boldsymbol{a m r} \boldsymbol{+}+\mathbf{4 0 0 b p}$ US \& DS & \\
amrR_Fullgene_F & TCCTGCCCTTCCTCGTAG \\
amrR_Fullgene_R & GCGGGCGGAAGAAACGGA \\
Site directed mutagenesis of $\boldsymbol{a m r R}$ T520C & \\
amrR_T520C_F & TGGTCCAGATCATCGGGCCGAAGATGCCTTC \\
amrR_T520C_R & GAAGGCATCTTCGGCCCGATGATCTGGACCA \\
Triplex efflux assay & \\
amrB_For_1 & TGTTCGCATGGGTGATCTCC \\
amrB_Rev_1 & GACCGATTCCTCGACGACCT \\
amrB_BHQ_Probe_1 & FAM-TGTTCATCATGCTGGGCGGCATC-BHQ1 \\
bpeB_For_1 & GCCGTCGATCCAGATCACC \\
bpeB_Rev_1 & TGCTCGATCACCTGCGTG \\
bpeB_BHQ_Probe_1 & JOE-TTCGGCGAAGACCGTCGAAGACAC-BHQ1 \\
bpeF_For_1 & TGACGTATCTGCGCAACTACG \\
bpeF_Rev_1 & TGCGGATCGAGCCACAC \\
bpef_BHQ_Probe_1 & Quasar 670-CTCATCAACGTGAAGGATCGCCTGTCAC-BHQ3 \\
\hline Abbreviations: BHQ, black hole quencher; US, upstream; DS, downstream.
\end{tabular}

Abbreviations: BHQ, black hole quencher; US, upstream; DS, downstream.

genome was performed to enable accurate variant annotation.

RNA extractions and cDNA synthesis. To determine expression levels of the three RND efflux pumps, B. pseudomallei clinical strains and mutants were grown to mid-log phase $\left(\mathrm{OD}_{600}=0.8\right.$ to 1$)$ in LB broth for RNA extraction unless otherwise stated. For two B. pseudomallei isogenic pairs (MSHR5864 and MSHR6755; MSHR3763 and MSHR4083) and an AmrR knockout (MSHR6755 $\Delta a m r R$ ), LB was supplemented with $0.25 \mu \mathrm{g} / \mathrm{mL}$ MEM (Sigma-Aldrich, Castle Hill, NSW, Australia); all other strains were grown in the absence of antibiotics. For the clinical B. pseudomallei pairs, biological replicates of total RNA were extracted using TRIzol according to the manufacturer's instructions (Thermo Fisher Scientific, Scoresby, VIC, Australia). RNA samples were treated with TURBO DNase (Thermo Fisher Scientific) prior to cDNA conversion using the QuantiTect Reverse Transcription Kit (Qiagen, Chadstone, VIC, Australia). Eradication of contaminating DNA prior to CDNA synthesis was confirmed with real-time PCR on the RNA extracts without the inclusion of reverse transcriptase to ensure no amplification. For the Bp82 mutants, biological triplicates of total RNA were extracted using the RNeasy Protect Bacteria kit according to the manufacturer's instructions (Qiagen), followed by DNase treatment (Fermentas, Waltham, MA, USA) and cDNA conversion using the SuperScript III First-Strand Synthesis SuperMix (Thermo Fisher Scientific).

Triplex assay design and quantitative PCR conditions. For the quantification of AmrAB-OprA, BpeAB-OprB and BpeEF-OprC expression, a triplex assay was designed to detect the RND transporter genes ( $a m r B, b p e B$ and $b p e F)$ of these efflux pumps. Primers and probes were designed using Primer Express software v3.0.1 (Applied Biosystems, Scoresby, VIC, Australia) and checked for specificity and binding efficiency 
Detection of efflux pump upregulation in B. pseudomallei

using MegaBLAST. NetPrimer (http://www.premierbiosoft.com/netprimer/) was used to guide oligo design to avoid primer dimer artefacts, with a within-assay cross- and selfdimer $(\Delta \mathrm{G})$ cut-off of -10 or higher deemed acceptable. Each assay was first performed and optimized in singleplex format, with probes initially being labeled with the FAM dye, before converting the assays to a triplex-compatible format using three spectrally distinct dyes: 5'-FAM-amrB-BHQ-1-3', 5'-JOE-bpeB-BHQ-1-3' and 5'-Quasar670-bpeFBHQ-1-3'. For $a m r B$ and $b p e B, 0.35 \mu \mathrm{M}$ of each primer was used (amrB-For_1, amrBRev_1, bpeB-For_1, bpeB-Rev_1) with $0.2 \mu \mathrm{M}$ of bpeF primers, together with $0.25 \mu \mathrm{M}$ of each Black Hole Quencher probe (LGC Biosearch Technologies, Petaluma, CA, USA) and 1 X TaqMan Environmental PCR Master Mix (Applied Biosystems).

Two conserved genes were used as controls for normalizing efflux pump gene expression. For all isolates, the TaqMan MGB probe-based mmsA (BPSS0619; also known as 266152) assay, which targets methylmalonate-semialdehyde dehydrogenase (31), was used. The mmsA PCRs were carried out using either 384-well optical plates on the QuantStudio 6 Flex Real-Time PCR system (Thermo Fisher Scientific), or in 96well optical plates using the CFX96 Touch Real-Time PCR detection system (Bio-Rad, Hercules, CA, USA). The limits of detection (LoD) and quantitation (LoQ) for the $m m s A$ assay have previously been determined as $4 \mathrm{fg}$, or 0.5 genomic equivalents (GEs), which is the equivalent of a single PCR template (31), making this assay attractive for low-concentration target quantification. For efflux pump gene normalization in eight Bp82 mutants, a $23 \mathrm{~S}$ rDNA assay was also used as described elsewhere (27). For $23 \mathrm{~S}$ qPCRs, $0.2 \mu \mathrm{M}$ of each primer was used with 2 X SYBR Select Mastermix (Thermo Fisher Scientific). The following conditions were used for thermocycling: enzyme activation for $2 \mathrm{~min}$ at $50^{\circ} \mathrm{C}$, initial denaturation at $95^{\circ} \mathrm{C}$ for $10 \mathrm{~min}$, followed by 45 cycles of denaturation at $95^{\circ} \mathrm{C}$ for $15 \mathrm{sec}$ and annealing for $1 \mathrm{~min}$ at $60^{\circ} \mathrm{C}$. The auto setting was used on each instrument when determining the threshold for each of the three assays in the triplex format.

Triplex assay performance. The performance of the triplex qPCR assay was tested across several criteria to determine the LoQ, LoD and linearity (efficiency) following previously published methods $(31,29)$. Briefly, genomic DNA from B. pseudomallei MSHR4420 was used as template due to this sample being reasonably concentrated (286 ng/ $\mu \mathrm{L}$ according to NanoDrop 2000 [Thermo Fisher Scientific] spectrophotometric analysis), of high quality and from a recent extraction. To establish the LoQ, LoD and linearity, 1:10 serial dilutions of $B$. pseudomallei MSHR4420 ranging from 40 to $4 \times 10^{-6}$ ng across eight replicates at each concentration were used as PCR template. Genomic equivalents (GEs) were calculated using an average molecular weight of $660 \mathrm{~g} / \mathrm{mol} / \mathrm{bp}$ and a 7.2 Mbp genome size.

Triplex qPCR assay use and statistics. The triplex qPCR assay was performed on the 15 clinical strains, and eight Bp82 laboratory-generated mutants that contained regulator mutations. Average relative expression values (calculated as both times (x) change and $\log _{2}$ fold change in expression) were calculated based on biological replicates or triplicates. The relative expression data was analyzed by two-way analysis of variance (ANOVA) and Tukey's multiple-comparison test using GraphPad Prism (GraphPad Software, Inc., La Jolla, CA). $p$ values of $<0.05$ were considered significant.

Availability of data and materials. WGS and accession numbers. Paired-end IIlumina WGS data for the clinical strains MSHR0052, MSHR0664, MSHR0937, MSHR5651, MSHR5654, MSHR0292, MSHR0293, MSHR6522, MSHR7929, MSHR3763, MSHR4083, MSHR5864, and MSHR6755 were previously generated to $~ 80-90 x$ coverage using the HiSeq2000 or HiSeq2500 platforms (Macrogen Inc., Geumcheon-gu, Seoul, Rep. of Korea). These reads have been deposited into the Sequence Read Archive database under 
bioRxiv preprint doi: https://doi.org/10.1101/301960; this version posted April 17, 2018. The copyright holder for this preprint (which was not certified by peer review) is the author/funder, who has granted bioRxiv a license to display the preprint in perpetuity. It is made available under aCC-BY-NC-ND 4.0 International license.

Webb et al.

\section{REFERENCES}

1. World Health Organization. Antimicrobial resistance: Global report on surveillance; 2014.

2. Centers for Disease Control and Prevention. Antibiotic resistance: Threats in the United States; 2013.

3. Dance DA, Wuthiekanun V, Chaowagul W, White NJ. The antimicrobial susceptibility of Pseudomonas pseudomallei. Emergence of resistance in vitro and during treatment. J Antimicrob Chemother 1989; 24(3):295-309. http://www.ncbi.nlm.nih.gov/pubmed/2681116.

4. Jenney AW, Lum G, Fisher DA, Currie BJ. Antibiotic susceptibility of Burkholderia pseudomallei from tropical northern Australia and implications for therapy of melioidosis. Int J Antimicrob Agents 2001; 17(2):10913. http://www.ncbi.nlm.nih.gov/pubmed/11165114.

5. Tribuddharat C, Moore RA, Baker P, Woods DE. Burkholderia pseudomallei class a beta-lactamase mutations that confer selective resistance against ceftazidime or clavulanic acid inhibition. Antimicrob Agents Chemother 2003; 47(7):2082-7. http://www.ncbi.nlm.nih.gov/pubmed/12821450.

6. Schweizer HP. Mechanisms of antibiotic resistance in Burkholderia pseudomallei: implications for treatment of melioidosis. Futur. Microbiol 2012; 7(12):1389-99. http://www.ncbi.nlm.nih.gov/pubmed/23231488.

7. JM W. Melioidosis: The most neglected tropical disease. mBiosphere 2017; 2017(22 09 2017). https://www.asm.org/index.php/mbiosphere/ item/5883-melioidosis-the-most-neglected-tropical-disease.
8. Limmathurotsakul D, Golding N, Dance DAB, Messina JP, Pigott DM, Moyes CL, Rolim DB, Bertherat E, Day NPJ, Peacock SJ, Hay SI. Predicted global distribution of Burkholderia pseudomallei and burden of melioidosis. Nat. Microbiol. 2016; 1(1):15008. http://dx.doi.org/10.1038/ nmicrobiol.2015.8.

9. Cheng AC, Fisher DA, Anstey NM, Stephens DP, Jacups SP, Currie BJ. Outcomes of patients with melioidosis treated with meropenem. Antimicrob Agents Chemother 2004; 48(5):1763-5. http://www.ncbi.nlm. nih.gov/pubmed/15105132.

10. Pitman MC, Luck T, Marshall CS, Anstey NM, Ward L, Currie BJ. Intravenous therapy duration and outcomes in melioidosis: A new treatment paradigm. PLoS Negl Trop Dis 2015; 9(3):e0003586. http: //www.ncbi.nlm.nih.gov/pubmed/25811783.

11. Piliouras $P$, Ulett GC, Ashhurst-Smith C, Hirst RG, Norton RE. A comparison of antibiotic susceptibility testing methods for cotrimoxazole with Burkholderia pseudomallei. Int J Antimicrob Agents 2002; 19(5):427-9. http://www.ncbi.nlm.nih.gov/pubmed/12007851.

12. Wuthiekanun V, Cheng AC, Chierakul W, Amornchai P, Limmathurotsakul D, Chaowagul W, Simpson AJ, Short JM, Wongsuvan G, Maharjan B, White NJ, Peacock SJ. Trimethoprim/sulfamethoxazole resistance in clinical isolates of Burkholderia pseudomallei. J Antimicrob Chemother 2005; 55(6):1029-31. http://www.ncbi.nlm.nih.gov/pubmed/15886263. 
bioRxiv preprint doi: https://doi.org/10.1101/301960; this version posted April 17, 2018. The copyright holder for this preprint (which was not certified by peer review) is the author/funder, who has granted bioRxiv a license to display the preprint in perpetuity. It is made available under aCC-BY-NC-ND 4.0 International license.

Detection of efflux pump upregulation in B. pseudomallei

13. Wuthiekanun V, Peacock SJ. Management of melioidosis. Expert. Rev Anti Infect Ther 2006; 4(3):445-55. http://www.ncbi.nlm.nih.gov/ pubmed/16771621.

14. Wuthiekanun V, Amornchai P, Saiprom N, Chantratita N, Chierakul W, Koh GC, Chaowagul W, Day NP, Limmathurotsakul D, Peacock SJ. Survey of antimicrobial resistance in clinical Burkholderia pseudomallei isolates over two decades in Northeast Thailand. Antimicrob Agents Chemother 2011; 55(11):5388-91. http://www.ncbi.nlm.nih.gov/pubmed/21876049.

15. Rakotondrasoa A, Issack MI, Garin B, Vallade E, Wattiau P, Allou N, Belmonte O, Bibi J, Price EP, Collard JM. Melioidosis in the western Indian Ocean and the importance of improving diagnosis, surveillance and molecular typing. Trop Med Infect Dis 2018; .

16. Price EP, Smith ML, Paxinos EE, Tallon LJ, Sadzewicz L, Sengamalay N, Baird RW, Currie BJ, Sarovich DS. Whole-Genome sequences of Burkholderia pseudomallei isolates exhibiting decreased meropenem susceptibility. Genome Announc. 2017; 5(14). http://www.ncbi.nlm.nih. gov/pubmed/28385830.

17. Sarovich DS, Webb JR, Pitman MC, Viberg LT, Mayo M, Baird RW, Robson $J M$, Currie BJ, Price EP. Raising the stakes: Loss of efflux-pump regulation decreases meropenem susceptibility in Burkholderia pseudomallei. Clin. Infect. Dis. 2018; http://dx.doi.org/10.1093/cid/ciy069.

18. Chan YY, Tan TM, Ong YM, Chua KL. BpeAB-OprB, a multidrug efflux pump in Burkholderia pseudomallei. Antimicrob Agents Chemother 2004; 48(4):1128-35. http://www.ncbi.nlm.nih.gov/pubmed/15047512.

19. Moore RA, DeShazer D, Reckseidler S, Weissman A, Woods DE. Effluxmediated aminoglycoside and macrolide resistance in Burkholderia pseudomallei. Antimicrob Agents Chemother 1999; 43(3):465-70. http: //www.ncbi.nlm.nih.gov/pubmed/10049252.

20. Kumar A, Chua KL, Schweizer HP. Method for regulated expression of single-copy efflux pump genes in a surrogate Pseudomonas aeruginosa strain: Identification of the BpeEF-OprC chloramphenicol and trimethoprim efflux pump of Burkholderia pseudomallei 1026b. Antimicrob Agents Chemother 2006; 50(10):3460-3. http://www.ncbi.nIm.nih. gov/pubmed/17005832.

21. Eswaran J, Koronakis E, Higgins MK, Hughes C, Koronakis V. Three's company: component structures bring a closer view of tripartite drug efflux pumps. Curr Opin Struct Biol 2004; 14(6):741-7. https://www.ncbi. nlm.nih.gov/pubmed/15582398.

22. Viberg LT, Sarovich DS, Kidd TJ, Geake JB, Bell SC, Currie BJ, Price EP. Within-host evolution of Burkholderia pseudomallei during chronic infection of seven Australasian cystic fibrosis patients. MBio 2017; 8(2). http://www.ncbi.nlm.nih.gov/pubmed/28400528.

23. Webb JR, Price EP, Currie BJ, Sarovich DS. Loss of methyltransferase function and increased efflux activity leads to doxycycline resistance in Burkholderia pseudomallei. Antimicrob Agents Chemother 2017; 61(6). http://www.ncbi.nlm.nih.gov/pubmed/28348161.

24. Podnecky NL, Rhodes KA, Mima T, Drew HR, Chirakul S, Wuthiekanun V, Schupp JM, Sarovich DS, Currie BJ, Keim P, Schweizer HP. Mechanisms of resistance to folate pathway inhibitors in Burkholderia pseudomallei: Deviation from the norm. MBio 2017; 8(5). http://www.ncbi.nlm.nih. gov/pubmed/28874476.

25. Price EP, Viberg LT, Kidd TJ, Bell SC, Currie BJ, Sarovich DS. Transcriptomic analysis of longitudinal Burkholderia pseudomallei infecting the cystic fibrosis lung. Under review 2018; .

26. Kumar A, Mayo M, Trunck LA, Cheng AC, Currie BJ, Schweizer HP. Expression of resistance-nodulation-cell-division efflux pumps in commonly used Burkholderia pseudomallei strains and clinical isolates from northern Australia. Trans R Soc Trop Med Hyg 2008; 102 Suppl 1:S145-51. http://www.ncbi.nlm.nih.gov/pubmed/19121677.

27. Mima T, Schweizer HP. The BpeAB-OprB efflux pump of Burkholderia pseudomallei $1026 \mathrm{~b}$ does not play a role in quorum sensing, viru- lence factor production, or extrusion of aminoglycosides but is a broadspectrum drug efflux system. Antimicrob Agents Chemother 2010; 54(8):3113-20. http://www.ncbi.nlm.nih.gov/pubmed/20498323.

28. Podnecky NL, Wuthiekanun V, Peacock SJ, Schweizer HP. The BpeEFOprC efflux pump is responsible for widespread trimethoprim resistance in clinical and environmental Burkholderia pseudomallei isolates. Antimicrob Agents Chemother 2013; 57(9):4381-6. http://www.ncbi.nlm. nih.gov/pubmed/23817379.

29. Araujo P. Key aspects of analytical method validation and linearity evaluation. J Chromatogr B Anal. Technol Biomed Life Sci 2009; 877(23):222434. http://www.ncbi.nlm.nih.gov/pubmed/18929516.

30. Svanvik N, Stahlberg A, Sehlstedt U, Sjoback R, Kubista M. Detection of PCR products in real time using light-up probes. Anal Biochem. 2000; 287(1):179-82. http://www.ncbi.nlm.nih.gov/pubmed/11078598.

31. Price EP, Dale JL, Cook JM, Sarovich DS, Seymour ML, Ginther JL, Kaufman EL, Beckstrom-Sternberg SM, Mayo M, Kaestli M, Glass MB, Gee JE, Wuthiekanun V, Warner JM, Baker A, Foster JT, Tan P, Tuanyok A, Limmathurotsakul D, Peacock SJ, Currie BJ, Wagner DM, Keim P, Pearson T. Development and validation of Burkholderia pseudomallei-specific real-time PCR assays for clinical, environmental or forensic detection applications. PLoS One 2012; 7(5):e37723. http://www.ncbi.nlm.nih.gov/ pubmed/22624061.

32. Wiersinga WJ, Currie BJ, Peacock SJ. Melioidosis. N Engl J Med 2012; 367(11):1035-44. https://www.ncbi.nlm.nih.gov/pubmed/22970946.

33. Sarovich DS, Price EP, Von Schulze AT, Cook JM, Mayo M, Watson LM, Richardson L, Seymour ML, Tuanyok A, Engelthaler DM, Pearson T, Peacock SJ, Currie BJ, Keim P, Wagner DM. Characterization of ceftazidime resistance mechanisms in clinical isolates of Burkholderia pseudomallei from Australia. PLoS One 2012; 7(2):e30789. http: //www.ncbi.nlm.nih.gov/pubmed/22363490.

34. Chantratita N, Rholl DA, Sim B, Wuthiekanun V, Limmathurotsakul $D$, Amornchai $P$, Thanwisai A, Chua HH, Ooi WF, Holden MT, Day NP, Tan P, Schweizer HP, Peacock SJ. Antimicrobial resistance to ceftazidime involving loss of penicillin-binding protein 3 in Burkholderia pseudomallei. Proc Natl Acad Sci U S A 2011; 108(41):17165-70. http://www.ncbi.nlm.nih.gov/pubmed/21969582.

35. Clinical and Laboratory Standards Institute. Performance standards for antimicrobial susceptibility testing; twenty-fifth informational supplement. Clin. Lab. Standards Institute, Wayne, PA 2015;

36. Malinga L, Brand J, Olorunju S, Stoltz A, van der Walt M. Molecular analysis of genetic mutations among cross-resistant second-line injectable drugs reveals a new resistant mutation in Mycobacterium tuberculosis. Diagn Microbiol Infect Dis 2016; 85(4):433-7. https: //www.ncbi.nlm.nih.gov/pubmed/27298046.

37. Toprak E, Veres A, Michel JB, Chait R, Hartl DL, Kishony R. Evolutionary paths to antibiotic resistance under dynamically sustained drug selection. Nat Genet. 2011; 44(1):101-5. https://www.ncbi.nlm.nih.gov/ pubmed/22179135.

38. Weinreich DM, Delaney NF, Depristo MA, Hartl DL. Darwinian evolution can follow only very few mutational paths to fitter proteins. Science 2006; 312(5770):111-4. https://www.ncbi.nlm.nih.gov/pubmed/ 16601193.

39. Maurin M. Real-time PCR as a diagnostic tool for bacterial diseases. Expert. Rev Mol Diagn 2012; 12(7):731-54. http://www.ncbi.nIm.nih.gov/ pubmed/23153240.

40. Abramson T, Kedem H, Relman DA. Proinflammatory and proapoptotic activities associated with Bordetella pertussis filamentous hemagglutinin. Infect Immun 2001; 69(4):2650-8. http://www.ncbi.nlm.nih.gov/ pubmed/11254631.

41. Rholl DA, Papp-Wallace KM, Tomaras AP, Vasil ML, Bonomo RA, Schweizer HP. Molecular investigations of PenA-mediated beta-lactam 
bioRxiv preprint doi: https://doi.org/10.1101/301960; this version posted April 17, 2018. The copyright holder for this preprint (which was not certified by peer review) is the author/funder, who has granted bioRxiv a license to display the preprint in perpetuity. It is made available under aCC-BY-NC-ND 4.0 International license.

Webb et al.

resistance in Burkholderia pseudomallei. Front Microbiol 2011; 2:139. http://www.ncbi.nlm.nih.gov/pubmed/21747814.

42. Sarovich DS, Price EP, Limmathurotsakul D, Cook JM, Von Schulze AT, Wolken SR, Keim P, Peacock SJ, Pearson T. Development of ceftazidime resistance in an acute Burkholderia pseudomallei infection. Infect Drug Resist. 2012; 5:129-32. http://www.ncbi.nlm.nih.gov/pubmed/22977307.

43. Hayden HS, Lim R, Brittnacher MJ, Sims EH, Ramage ER, Fong C, Wu Z, Crist E, Chang J, Zhou Y, Radey M, Rohmer L, Haugen E, Gillett W, Wuthiekanun V, Peacock SJ, Kaul R, Miller SI, Manoil C, Jacobs MA. Evolution of Burkholderia pseudomallei in recurrent melioidosis. PLoS One 2012; 7(5):e36507. https://www.ncbi.nlm.nih.gov/pubmed/22615773.

44. Teran W, Felipe A, Segura A, Rojas A, Ramos JL, Gallegos MT. Antibioticdependent induction of Pseudomonas putida DOT-T1E TtgABC efflux pump is mediated by the drug binding repressor TtgR. Antimicrob Agents Chemother 2003; 47(10):3067-72. http://www.ncbi.nlm.nih.gov/ pubmed/14506010.

45. Schumacher MA, Brennan RG. Deciphering the molecular basis of multidrug recognition: Crystal structures of the Staphylococcus aureus multidrug binding transcription regulator QacR. Res Microbiol 2003; 154(2):69-77. http://www.ncbi.nlm.nih.gov/pubmed/12648720.

46. Buckingham L, Flaws ML. Molecular diagnostics: Fundamentals, methods, \& clinical applications. Philadelphia, Pa, USA: F.A. Davis; 2007.

47. Beld M, Minnaar R, Weel J, Sol C, Damen M, van der Avoort H, Wertheimvan Dillen P, van Breda A, Boom R. Highly sensitive assay for detection of enterovirus in clinical specimens by reverse transcription-PCR with an armored RNA internal control. J Clin Microbiol 2004; 42(7):3059-64. https://www.ncbi.nlm.nih.gov/pubmed/15243060.

48. Kajiura LN, Stewart SD, Dresios J, Uyehara CF. Simultaneous Extraction of Viral and Bacterial Nucleic Acids for Molecular Diagnostic Applications. J Biomol Tech 2015; 26(4):118-24. https://www.ncbi.nlm.nih.gov/ pubmed/26543438.

49. Crisan A, McKee G, Munzner T, Gardy JL. Evidence-based design and evaluation of a whole genome sequencing clinical report for the reference microbiology laboratory. PeerJ 2018; 6:e4218. https: //www.ncbi.nlm.nih.gov/pubmed/29340235.

50. Propst KL, Mima T, Choi KH, Dow SW, Schweizer HP. A Burkholderia pseudomallei deltapurM mutant is avirulent in immunocompetent and immunodeficient animals: candidate strain for exclusion from selectagent lists. Infect Immun 2010; 78(7):3136-43. http://www.ncbi.nIm.nih. gov/pubmed/20404077.

51. Lopez CM, Rholl DA, Trunck LA, Schweizer HP. Versatile dual-technology system for markerless allele replacement in Burkholderia pseudomallei. Appl Environ Microbiol 2009; 75(20):6496-503. http://www.ncbi.nlm.nih. gov/pubmed/19700544.

52. Liss L. New M13 host: DH5 F' competent cells. Focus 1987; 9(13).

53. Clinical and Laboratory Standards Institute. Methods for antimicrobial dilution and disk susceptibility testing of infrequently isolated or fastidious bacteria approved guideline. Clin. Lab. Standards Institute, Wayne, PA 2015; 3rd ed. CLSI, Wayne, PA.
54. Crowe A, McMahon N, Currie BJ, Baird RW. Current antimicrobial susceptibility of first-episode melioidosis Burkholderia pseudomallei isolates from the Northern Territory, Australia. Int J Antimicrob Agents 2014; 44(2):160-2. http://www.ncbi.nlm.nih.gov/pubmed/24924662.

55. Maloney S, Engler C, Norton R. Epidemiological cut-off value of clinical isolates of Burkholderia pseudomallei from Northern Queensland to meropenem, ceftazidime, trimethoprim/sulfamethoxazole and doxycycline by the microbroth dilution method. J Glob Antimicrob Resist. 2017; 10:291-294. http://www.ncbi.nlm.nih.gov/pubmed/28729203.

56. Choi KH, Mima T, Casart Y, Rholl D, Kumar A, Beacham IR, Schweizer HP. Genetic tools for select-agent-compliant manipulation of Burkholderia pseudomallei. Appl Environ Microbiol 2008; 74(4):1064-75. http: //www.ncbi.nlm.nih.gov/pubmed/18156318.

57. Johnson SL, Baker AL, Chain PS, Currie BJ, Daligault HE, Davenport KW, Davis CB, Inglis TJ, Kaestli M, Koren S, Mayo M, Merritt AJ, Price EP, Sarovich DS, Warner J, Rosovitz MJ. Whole-genome sequences of 80 environmental and clinical isolates of Burkholderia pseudomallei. Genome Announc. 2015; 3(1). http://www.ncbi.nlm.nih.gov/pubmed/25676747.

58. Li H, Durbin R. Fast and accurate short read alignment with burrowswheeler transform. Bioinformatics 2009; 25(14):1754-60. http://www. ncbi.nlm.nih.gov/pubmed/19451168.

59. Li H, Handsaker B, Wysoker A, Fennell T, Ruan J, Homer N, Marth G, Abecasis $G$, Durbin R. The sequence alignment/map format and SAMtools. Bioinformatics 2009; 25(16):2078-9. http://www.ncbi.nIm.nih.gov/ pubmed/19505943.

60. McKenna A, Hanna M, Banks E, Sivachenko A, Cibulskis K, Kernytsky A, Garimella K, Altshuler D, Gabriel S, Daly M, DePristo MA. The genome analysis toolkit: A map reduce framework for analyzing nextgeneration DNA sequencing data. Genome Res 2010; 20(9):1297-303. http://www.ncbi.nlm.nih.gov/pubmed/20644199.

61. Cingolani P, Platts A, Wang le L, Coon M, Nguyen T, Wang L, Land SJ, Lu X, Ruden DM. A program for annotating and predicting the effects of single nucleotide polymorphisms, SnpEff: SNPs in the genome of Drosophila melanogaster strain w1118; iso-2; iso-3. Fly (Austin) 2012; 6(2):80-92. http://www.ncbi.nlm.nih.gov/pubmed/22728672.

62. Sarovich DS, Price EP. SPANDx: A genomics pipeline for comparative analysis of large haploid whole genome re-sequencing datasets. BMC Res Notes 2014; 7:618. http://www.ncbi.nlm.nih.gov/pubmed/25201145.

63. Holden MT, Titball RW, Peacock SJ, Cerdeno-Tarraga AM, Atkins T, Crossman LC, Pitt T, Churcher C, Mungall K, Bentley SD, Sebaihia M, Thomson NR, Bason N, Beacham IR, Brooks K, Brown KA, Brown NF, Challis GL, Cherevach I, Chillingworth T, Cronin A, Crossett B, Davis P, DeShazer D, Feltwell T, Fraser A, Hance Z, Hauser H, Holroyd S, Jagels K, Keith KE, Maddison M, Moule S, Price C, Quail MA, Rabbinowitsch E, Rutherford K, Sanders M, Simmonds M, Songsivilai S, Stevens K, Tumapa S, Vesaratchavest M, Whitehead S, Yeats C, Barrell BG, Oyston PC, Parkhill J. Genomic plasticity of the causative agent of melioidosis, Burkholderia pseudomallei. Proc Natl Acad Sci U S A 2004; 101(39):14240-5. http://www.ncbi.nlm.nih.gov/pubmed/15377794. 